\title{
Efeitos da temperatura no desenvolvimento, fecundidade e longevidade de Gargaphia torresi Lima (Hemiptera, Tingidae)
}

\author{
Carlos Alberto Domingues da Silva ${ }^{1}$
}

${ }^{1}$ EMBRAPA - Centro Nacional de Pesquisa de Algodão (CNPA). Caixa Postal 174, 58107-720 Campina Grande-PB, Brasil. Endereço eletrônico: cads@insecta.ufv.br

\begin{abstract}
Aвstract. Effects of temperature on the development, fecundity, and longevity of Gargaphia torresi Lima (Hemiptera, Tingidae). The development of Gargaphia torresi Lima on cotton leaves was studied in bioclimatic chambers at 20, 23, 25,28 and $30^{\circ} \mathrm{C}$, relative humidity of $70 \pm 10 \%$, and a $12 \mathrm{~h}: 12 \mathrm{~h}(\mathrm{~L}: \mathrm{D})$ photoperiod. The incubation period varied from 11 $\left(20^{\circ} \mathrm{C}\right)$ to $4\left(30^{\circ} \mathrm{C}\right)$ days; nymphal stage of individuals that originated females and males varied from $20.8\left(20^{\circ} \mathrm{C}\right)$ to 8.3 $\left(30^{\circ} \mathrm{C}\right)$ days and from $20.8\left(20^{\circ} \mathrm{C}\right)$ to $8.6\left(30^{\circ} \mathrm{C}\right)$ days, respectively. Development of immature stages for individuals that originated females and males varied from $31.8\left(20^{\circ} \mathrm{C}\right)$ to $12.3\left(30^{\circ} \mathrm{C}\right)$ days and from $31.5\left(20^{\circ} \mathrm{C}\right)$ to $12.6\left(30^{\circ} \mathrm{C}\right)$ days, respectively. The values of longevity and fecundity were inversely proportional with increase of the temperature. The thermal constant $(\mathrm{K})$ and theoretical lower threshold temperature of development $(\mathrm{Tb})$ were estimated by Reamur law and intercession method of the $\mathrm{X}$, respectively. The theoretical lower threshold temperature of development for individuals that originated adult females and males were $13.9^{\circ} \mathrm{C}$ and $13.6^{\circ} \mathrm{C}$, respectively. The immature stages for individuals that originated adult females and males required 209.4 and 220.3 degrees-days from oviposition to adult emergence, respectively.
\end{abstract}

Keywords. Biology; Gargaphia torresi; Hemiptera; temperature; threshold temperature.

Resumo. O desenvolvimento de Gargaphia torresi Lima em folhas de algodão foi estudado em câmara climatizada a 20, $23,25,28$ e $30^{\circ} \mathrm{C}$, umidade relativa de $70 \pm 10 \%$ e fotofase de $12 \mathrm{~h}$. O período de incubação variou de $11\left(20^{\circ} \mathrm{C}\right)$ a $4\left(30^{\circ} \mathrm{C}\right)$ dias; o estágio ninfal de indivíduos que originaram fêmeas e machos variou de $20,8\left(20^{\circ} \mathrm{C}\right)$ a $8,3\left(30^{\circ} \mathrm{C}\right)$ dias e de 28,8 $\left(20^{\circ} \mathrm{C}\right)$ e $8,6\left(30^{\circ} \mathrm{C}\right)$ dias, respectivamente. $\mathrm{O}$ desenvolvimento dos estágios imaturos para indivíduos que originaram fêmeas e machos variou de $31,8\left(20^{\circ} \mathrm{C}\right)$ a $12,3\left(30^{\circ} \mathrm{C}\right)$ dias e de $31,5\left(20^{\circ} \mathrm{C}\right)$ a $12,6\left(30^{\circ} \mathrm{C}\right)$ dias, respectivamente. Os valores de longevidade e fecundidade foram inversamente proporcionais ao aumento da temperatura. As exigências térmicas $(\mathrm{K})$ e temperaturas-base $(\mathrm{Tb})$ foram estimadas para cada um dos estágios imaturos e de ovo à emergência de adultos (machos e fêmeas) de acordo com a Lei de Reamur e com o método de intercecção de X, respectivamente. Os estágios imaturos para indivíduos que originaram fêmeas e machos adultos requereram 209,4 e 220,3 graus-dia da oviposição à emergência do adulto, respectivamente, acima dos limites inferiores de 13,9 e $13,6^{\circ} \mathrm{C}$.

Palavras-Chave. Biologia; Gargaphia torresi; Hemiptera; temperatura; temperatura-base.

Gargaphia torresi Lima é um inseto que se encontra amplamente distribuído em diversos estados brasileiros atacando, além do algodoeiro, outras plantas, como: caupi (Moraes \& Ramalho 1980; Moraes 1981, 1982), batata doce, milho (Drake \& Hambleton 1934), Croton sp., Triumfetta $\mathbf{s p .}$ (Drake \& Hambleton 1934; Monte 1939) e Sida sp. (Monte 1939). Na cultura do algodão, G. torresi pode alcançar níveis populacionais bastante elevados principalmente durante períodos secos e quentes do ano (Bleicher \& Freire 1980).

O hábito sugador do inseto faz com que as plantas atacadas apresentem manchas brancas, cloróticas nas folhas, que correspondem, na face inferior, aos locais onde se situam as colônias. Dependendo da intensidade do ataque, as plantas ficam raquíticas, podendo até morrer (Moraes 1981; SiLva \& BARBOSA 1986). Os danos são observados primeiramente nas folhas do terço inferior da planta (Bleicher \& Freire 1980) e, de acordo com VIEIRA et al. (1991), as perdas na produção de algodoeiro herbáceo podem variar de 19,83 a 54,70\%.

Apesar da grande importância atribuída a esse percevejo, pouco se conhece sobre os efeitos da temperatura sobre o seu desenvolvimento biológico. Os estudos sobre aspectos biológicos de tingídeos do gênero Gargaphia têm sido conduzidos com as espécies G. decoris (OlCKers 2000), G. sanchezi (Schoonhoven et al. 1975), G. solani (Kearns \& Yamamoto 1982; Tallamy \& Denno 1981; Tallamy 1985; Tallamy \& Horton 1990; Aldrich et al. 1991; Hardin \& Tallamy 1992; Monaco et al. 1998) e G. tiliae (Sheeley \& Yonke 1977; Hardin \& Tallamy 1992). Nesses estudos, a maioria das informações geradas tem considerado apenas aspectos biológicos relacionados ao comportamento de fêmeas em relação ao local de postura e defesa da progênie.

Assim, a geração de conhecimentos sobre a bionomia e necessidades térmicas desse inseto são de fundamental importância para se construir modelos matemáticos capazes de prever sua ocorrência, auxiliando na adoção de medidas de monitoramento e controle. O objetivo do presente trabalho foi quantificar os efeitos da temperatura no desenvolvimento, fecundidade e longevidade, de G. torresi $i$ as exigências térmicas para as diferentes fases de seu ciclo biológico em algodoeiro. 
Tabela I. Sobrevivência e duração média de desenvolvimento das fases de ovo, de ninfa e de ovo à emergência de adultos de Gargaphia torresi, às temperaturas de 20 a $30^{\circ} \mathrm{C}$, umidade relativa do ar de $70 \pm 10$ e fotofase de $12 \mathrm{~h}$. Campina Grande, 1999.

\begin{tabular}{|c|c|c|c|c|c|c|c|}
\hline \multirow[t]{2}{*}{ Estágio } & \multirow{2}{*}{$\begin{array}{c}\text { Temperatura } \\
\left({ }^{\circ} \mathrm{C}\right)\end{array}$} & \multirow[t]{2}{*}{ Indivíduos } & \multirow{2}{*}{$\begin{array}{c}\text { Sobrevivência } \\
(\%)\end{array}$} & \multicolumn{4}{|c|}{ Duração (dia) \pm erro padrão ${ }^{(1)}$} \\
\hline & & & & Fêmea & $\mathrm{n}$ & Macho & $\mathrm{n}$ \\
\hline \multirow[t]{5}{*}{ Ovo } & 20 & 39 & 97,5 & $11,0 \pm 0,0$ & 16 & $11,0 \pm 0,0^{\mathrm{N} . \mathrm{S}}$ & 19 \\
\hline & 23 & 40 & 100,0 & $\begin{array}{l}11,0 \pm 0,0 \\
8,0 \pm 0,0\end{array}$ & 16 & $8,0 \pm 0,0^{\text {N.S }}$ & 21 \\
\hline & 25 & 40 & 100,0 & $7,0 \pm 0,0$ & 19 & $7,0 \pm 0,0^{\text {N.S }}$ & 16 \\
\hline & 28 & 40 & 100,0 & $5,0 \pm 0,0$ & 24 & $5,0 \pm 0,0^{\mathrm{N} . \mathrm{S}}$ & 12 \\
\hline & 30 & 39 & 97,5 & $4,0 \pm 0,0$ & 15 & $4,0 \pm 0,0^{\mathrm{N} . \mathrm{S}}$ & 20 \\
\hline \multirow[t]{5}{*}{ Ninfal } & 20 & 35 & 89,7 & $20,8 \pm 1,3$ & 16 & $20,8 \pm 0,9^{\mathrm{N} . \mathrm{S}}$ & 19 \\
\hline & 23 & 37 & 92,5 & $14,6 \pm 1,1$ & 16 & $14,7 \pm 1,2^{\text {N.S }}$ & 21 \\
\hline & 25 & 35 & 87,5 & $13,7 \pm 0,6$ & 19 & $14,6 \pm 0,7^{*}$ & 16 \\
\hline & 28 & 36 & 90,0 & $10,5 \pm 0,7$ & 24 & $11,0 \pm 1,1^{*}$ & 12 \\
\hline & 30 & 35 & 89,7 & $8,3 \pm 0,7$ & 15 & $8,6 \pm 0,5^{\mathrm{N} . \mathrm{S}}$ & 20 \\
\hline \multirow[t]{5}{*}{ Ovo a adulto } & 20 & 35 & 87,5 & $31,9 \pm 1,3$ & 13 & $31,5 \pm 1,2^{\mathrm{N} . S}$ & 16 \\
\hline & 23 & 37 & 92,5 & $22,6 \pm 1,1$ & 16 & $22,7 \pm 1,2^{\text {N.S }}$ & 21 \\
\hline & 25 & 35 & 87,5 & $20,7 \pm 0,6$ & 19 & $21,6 \pm 0,7^{*}$ & 16 \\
\hline & 28 & 36 & 90,0 & $15,5 \pm 0,7$ & 24 & $16,0 \pm 1,1^{*}$ & 12 \\
\hline & 30 & 35 & 87,5 & $12,3 \pm 0,7$ & 15 & $12,6 \pm 0,5^{\mathrm{N} . \mathrm{S}}$ & 20 \\
\hline
\end{tabular}

${ }^{(1)}$ NS Não há diferença significativa na duração de desenvolvimento entre fêmea e macho pelo teste "t" $(\mathrm{P}=0,05)$; *Há diferença significativa na duração de desenvolvimento entre fêmea e macho pelo teste " $\mathrm{t}$ " $(\mathrm{P}=0,05)$; n: Número de indivíduos que atingiram o estágio adulto.

\section{MATERIAL E MÉTODOS}

O trabalho foi conduzido no laboratório de Entomologia da EMBRAPA - Centro Nacional de Pesquisa de Algodão, em Campina Grande, Paraíba, às temperaturas de 20, 23, 25, 28 e $30^{\circ} \mathrm{C}$, umidade relativa de $70 \pm 10 \%$ e fotofase de $12 \mathrm{~h}$. Os insetos utilizados neste estudo foram obtidos da criação-estoque mantida no referido laboratório.

Para obtenção dos ovos, 200 adultos de G. torresi foram transferidos da criação-estoque para folhas de algodão cultivar CNPA ITA 90 para efetuarem posturas. Vinte e quatro horas após a transferência, as fêmeas foram eliminadas e as folhas pontuadas com tinta azul, para marcação dos ovos a serem observados. Para cada temperatura testada, 40 ovos foram observados diariamente com auxílio de um microscópio estereoscópico até a emergência de ninfas de primeiro ínstar, e para que as folhas se mantivessem túrgidas, os pecíolos foram inseridos, isoladamente, dentro de frascos de vidro $(4,5 \mathrm{~cm} \mathrm{x}$ $9,0 \mathrm{~cm}$ ), preenchidos com água destilada.

Ninfas recém-emergidas foram transferidas isoladamente para tubos de PVC $(2,5 \mathrm{~cm} \times 2,0 \mathrm{~cm})$ fechados na base. No interior dos tubos foi colocado um chumaço de algodão umedecido e, sobre este, um disco de algodão medindo $2,4 \mathrm{~cm}$ de diâmetro, tomado da face ventral da folha da cultivar CNPA ITA 90. Posteriormente, as unidades de criação foram tampadas com uma película de filme plástico transparente e mantidas em estufa incubadora B.O.D., até os insetos atingirem o estágio adulto.
As observações biológicas foram realizadas diariamente, sempre às 8:00 e às 16:00 horas, com auxílio de um microscópio estereoscópico.

Para cada temperatura quantificaram-se as seguintes variáveis biológicas: fase de ovo (duração e viabilidade), ninfa (duração total, duração de cada ínstar, número de ínstares e sobrevivência); período de ovo à emergência de adultos machos e fêmeas, que foram sexados de acordo com a genitália. $\mathrm{Na}$ fase adulta avaliaram-se os períodos de pré-oviposição, oviposição, pós-oviposição, longevidade e fecundidade. As diferenças nas durações de desenvolvimento entre machos e fêmeas de G. torresi, foram comparadas pelo teste "t". Para se determinar a fecundidade e a razão sexual da progênie em cada temperatura, os adultos foram separados em casais, sendo os machos recém-emergidos transferidos isoladamente, para novas arenas contendo uma fêmea recém-emergida. O número de ovos depositados nessas arenas foi quantificado diariamente, até às 16:00 horas. As exigências térmicas $(\mathrm{K}) \mathrm{e}$ temperaturas-base $(\mathrm{Tb})$ foram estimadas para cada um dos estágios imaturos e de ovo à emergência de adultos (machos e fêmeas), de acordo com Lei de Reamur e com o método da intersecção de $\mathrm{X}$, respectivamente.

\section{RESULTADOS E DISCUSSÃO}

As fêmeas geralmente fazem postura na face inferior das folhas, em meio a seus excrementos, ficando os ovos depositados na superfície das folhas e parcialmente embutidos 
Tabela II.Sobrevivência e duração média de desenvolvimento de cada um dos estágios ninfais de Gargaphia torresi, às temperaturas de 20 a $30^{\circ} \mathrm{C}$, umidade relativa do ar de $70 \pm 10$ e fotofase de 12h. Campina Grande, 1999.

\begin{tabular}{|c|c|c|c|c|c|c|c|}
\hline \multirow{2}{*}{$\begin{array}{l}\text { Estágio } \\
\text { Ninfal }\end{array}$} & \multirow{2}{*}{$\begin{array}{c}\text { Temperatura } \\
\left({ }^{\circ} \mathrm{C}\right)\end{array}$} & \multirow[t]{2}{*}{ Indivíduos } & \multirow{2}{*}{$\begin{array}{c}\text { Sobrevivência } \\
(\%)\end{array}$} & \multicolumn{4}{|c|}{ Duração (dia) \pm erro padrão ${ }^{(1)}$} \\
\hline & & & & Fêmea & $\mathrm{n}$ & Macho & $\mathrm{n}$ \\
\hline \multirow[t]{5}{*}{$1^{\circ}$ ínstar } & 20 & 39 & 94,9 & $4,7 \pm 0,4$ & 16 & $4,5 \pm 0,4^{\mathrm{N} . \mathrm{S}}$ & 19 \\
\hline & 23 & 40 & 100,0 & $2,3 \pm 0,4$ & 16 & $2,3 \pm 0,4^{\mathrm{N} . \mathrm{S}}$ & 21 \\
\hline & 25 & 40 & 92,5 & $2,9 \pm 0,3$ & 19 & $3,2 \pm 0,5^{*}$ & 16 \\
\hline & 28 & 40 & 92,5 & $2,0 \pm 0,1$ & 24 & $2,1 \pm 0,2^{\mathrm{N} . \mathrm{S}}$ & 12 \\
\hline & 30 & 39 & 97,4 & $2,0 \pm 0,4$ & 15 & $2,0 \pm 0,6^{\mathrm{N} . \mathrm{S}}$ & 20 \\
\hline \multirow[t]{5}{*}{$2^{\circ}$ ínstar } & 20 & 37 & 100,0 & $3,0 \pm 0,7$ & 16 & $2,5 \pm 0,5^{*}$ & 19 \\
\hline & 23 & 40 & 100,0 & $2,4 \pm 0,5$ & 16 & $2,5 \pm 0,4^{\mathrm{N} . \mathrm{S}}$ & 21 \\
\hline & 25 & 37 & 100,0 & $2,0 \pm 0,4$ & 19 & $2,3 \pm 0,3^{*}$ & 16 \\
\hline & 28 & 37 & 100,0 & $1,4 \pm 0,3$ & 24 & $1,4 \pm 0,2^{\mathrm{N} . \mathrm{S}}$ & 12 \\
\hline & 30 & 38 & 100,0 & $1,2 \pm 0,3$ & 15 & $1,2 \pm 0,4^{\mathrm{N} . \mathrm{S}}$ & 20 \\
\hline \multirow[t]{5}{*}{$3^{\circ}$ ínstar } & 20 & 37 & 97,3 & $3,1 \pm 0,6$ & 16 & $3,3 \pm 0,5^{\mathrm{N} . \mathrm{S}}$ & 16 \\
\hline & 23 & 40 & 97,5 & $2,3 \pm 0,5$ & 16 & $2,7 \pm 0,3^{*}$ & 21 \\
\hline & 25 & 37 & 97,3 & $2,1 \pm 0,4$ & 19 & $2,3 \pm 0,3^{\mathrm{N} . \mathrm{S}}$ & 16 \\
\hline & 28 & 37 & 100,0 & $1,9 \pm 0,4$ & 24 & $2,0 \pm 0,5^{\mathrm{N} . \mathrm{S}}$ & 12 \\
\hline & 30 & 38 & 100,0 & $0,9 \pm 0,2$ & 15 & $0,8 \pm 0,3^{*}$ & 20 \\
\hline \multirow[t]{5}{*}{$4^{\circ}$ ínstar } & 20 & 36 & 97,2 & $3,7 \pm 0,6$ & 16 & $4,2 \pm 0,7^{\mathrm{N} . \mathrm{S}}$ & 16 \\
\hline & 23 & 39 & 94,9 & $3,0 \pm 0,3$ & 16 & $2,7 \pm 0,3^{\mathrm{N} . \mathrm{S}}$ & 21 \\
\hline & 25 & 36 & 97,2 & $2,7 \pm 0,4$ & 19 & $2,3 \pm 0,4^{\mathrm{N} . \mathrm{S}}$ & 16 \\
\hline & 28 & 37 & 97,3 & $1,8 \pm 0,3$ & 24 & $2,0 \pm 0,7^{\mathrm{N} . \mathrm{S}}$ & 12 \\
\hline & 30 & 38 & 92,1 & $1,4 \pm 0,5$ & 15 & $1,7 \pm 0,3^{\mathrm{N} . \mathrm{S}}$ & 20 \\
\hline \multirow[t]{5}{*}{$5^{\circ}$ ínstar } & 20 & 35 & 100,0 & $6,3 \pm 0,4$ & 16 & $6,4 \pm 0,6^{\mathrm{N} . S}$ & 16 \\
\hline & 23 & 37 & 100,0 & $4,7 \pm 0,5$ & 16 & $4,8 \pm 0,8^{\mathrm{N} . \mathrm{S}}$ & 21 \\
\hline & 25 & 35 & 100,0 & $4,1 \pm 0,2$ & 19 & $4,2 \pm 0,4^{\mathrm{N} . \mathrm{S}}$ & 16 \\
\hline & 28 & 36 & 100,0 & $3,4 \pm 0,5$ & 24 & $3,6 \pm 0,5^{*}$ & 12 \\
\hline & 30 & 35 & 100,0 & $2,9 \pm 0,4$ & 15 & $2,8 \pm 0,4^{\mathrm{N} . \mathrm{S}}$ & 20 \\
\hline
\end{tabular}

(1)NS: não há diferença significativa na duração de desenvolvimento entre fêmea e macho pelo teste "t" ( $\mathrm{P}=0,05)$; *: há diferença significativa na duração de desenvolvimento entre fêmea e macho pelo teste " $\mathrm{t}$ " $(\mathrm{P}=0,05)$; n: número de indivíduos que atingiram o estágio adulto.

no parênquima foliar, como descrito por Costa LiMA (1940). Os ovos com formato de cone, apresentam a porção apical mais afilada e achatada, medindo cerca de $2 \mathrm{~mm}$ de comprimento e coloração variável do branco ao creme; são depositados isoladamente, porém existe tendência para que novos ovos sejam colocados nos locais onde já existem posturas. A viabilidade dos ovos de $G$. torresi foi elevada nas cinco temperaturas estudadas, cujas porcentagens variaram de 97,5 $\left(20\right.$ e $\left.30^{\circ} \mathrm{C}\right)$ a $100 \%\left(23,25\right.$ e $\left.28^{\circ} \mathrm{C}\right)$ (Tabela I). A temperatura afetou a duração do período de incubação, promovendo uma variação de $4\left(30^{\circ} \mathrm{C}\right)$ a 11 dias $\left(20^{\circ} \mathrm{C}\right)$. A $25^{\circ} \mathrm{C}$, o período de incubação de $G$. torresi, de 7 dias, foi superior aos 5,4 dias observado por SCHOONHOVEn et al. (1975) para G. sanchezi em folhas de feijoeiro e inferior aos períodos de incubação de 9,3 (Rogers 1977) e 12 dias (Neal JR. \& Douglass 1990) de Coryntucha morrilli e aos 12 (BRAMAn et al. 1992) e 13 dias
(NEAL JR. \& Douglass 1988) de Stephanitis pyrioides, em folhas da azaleia.

A fase ninfal apresentou alta sobrevivência nas cinco temperaturas estudadas, demonstrando que ninfas de $G$. torresi podem sobreviver normalmente dentro desse gradiente de temperatura. Essa fase é caracterizada por apresentar cinco ínstares, que se diferenciam em tamanho e no comprimento dos espinhos dorsais e, a partir do $3^{\circ}$ ínstar, pela presença e comprimento das tecas alares. As porcentagens de sobrevivência de $G$. torresi durante o primeiro, terceiro e quarto ínstares nas cinco temperaturas estudadas, variaram, respectivamente, de 92,5 a $100 \%$, de 97,3 a $100 \%$ e de 92,1 a $97,2 \%$, constatando-se $100 \%$ de sobreviventes no segundo e quinto ínstares (Tabela II).

A duração do estágio de ninfa de $G$. torresi variou de 20,8 $\left(20^{\circ} \mathrm{C}\right)$ a $8,3 \operatorname{dias}\left(30^{\circ} \mathrm{C}\right)$ para adultos que originaram machos e 
Tabela III. Períodos (dias) de pré-oviposicão, oviposição, pós-oviposicão, longevidade e de fecundidade de Gargaphia torresi, às temperaturas de 20 a $30^{\circ} \mathrm{C}$, umidade relativa do ar $70 \pm 10$ e fotofase de $12 \mathrm{~h}$. Campina Grande, 1999.

\begin{tabular}{cccccccc}
\hline $\begin{array}{c}\text { Temperatura } \\
\left({ }^{\circ} \mathrm{C}\right)\end{array}$ & sexo & $\mathrm{n}$ & Pré-oviposição & Oviposição & Pós-oviposição & Longevidade & Fecundidade \\
\hline 20 & Fêmea & 13 & $8,7 \pm 2,3$ & $61,9 \pm 21,5$ & $7,5 \pm 7,9$ & $83,4 \pm 21,0$ & $219,3 \pm 69,1$ \\
& Macho & 16 & - & - & - & $120,9 \pm 42,3$ & - \\
23 & Fêmea & 16 & $6,3 \pm 1,1$ & $49,0 \pm 04,8$ & $5,5 \pm 3,1$ & $67,2 \pm 11,1$ & $199,8 \pm 57,4$ \\
& Macho & 22 & - & - & - & $96,1 \pm 30,1$ & - \\
25 & Fêmea & 17 & $5,6 \pm 1,6$ & $34,9 \pm 17,2$ & $3,4 \pm 0,0$ & $44,9 \pm 18,9$ & $183,1 \pm 95,9$ \\
& Macho & 15 & - & - & - & $63,5 \pm 22,0$ & - \\
28 & Fêmea & 22 & $4,4 \pm 1,8$ & $18,2 \pm 04,7$ & $2,5 \pm 3,5$ & $27,3 \pm 10,2$ & $103,1 \pm 60,5$ \\
& Macho & 14 & - & - & - & $50,2 \pm 27,5$ & - \\
30 & Fêmea & 14 & $4,1 \pm 2,5$ & $09,7 \pm 09,4$ & $1,6 \pm 1,8$ & $20,1 \pm 08,3$ & $61,0 \pm 44,1$ \\
& Macho & 19 & - & - & - & $30,1 \pm 15,2$ & - \\
\hline
\end{tabular}

fêmeas (Tabela I). Dentro desse mesmo gradiente de temperatura, foram registrados resultados semelhantes para a duração de desenvolvimento do estágio ninfal de Coryntucha cydoniae (Neal JR. \& Douglass 1990) e S. pyrioides (NEAL JR. \& Douglass 1988). Por outro lado, a $25^{\circ} \mathrm{C}$, a duração dos ínstares de $G$. torresi foi superior à registrada para G. sanchesi (SchoONHOVEn et al. 1975).

O tempo de desenvolvimento de $G$. torresi variou em cada ínstar, entre ínstares e entre os sexos (Tabelas I e II) de acordo com a temperatura. Dentre os ínstares, as menores durações foram observadas nos segundo e terceiro, enquanto que a maior duração foi observada no quinto, concordando com resultados anteriores obtidos para diversas espécies e gêneros da família Tingidae, incluindo Gargaphia (Schoonhoven et al. 1975), Stephanitis (Dunbar 1974; Neal JR. \& Douglass 1988; Braman et al. 1992) e Corynthucha (Rogers 1977; Silverman \& Goeden 1979; Horn et al. 1980; Vogt \& MCPHERson 1986; Braman \& Pendley 1993; Neal Jr. \& Douglass 1990). É provável que essa maior duração de desenvolvimento de ninfas de quinto ínstar esteja relacionada ao aumento no tempo de consumo e alocação de energia para promover modificações na forma e na constituição do inseto, necessárias para a ninfa emergir em adulto. Com exceção das ninfas de primeiro $\left(20^{\circ} \mathrm{C}\right)$, segundo $\left(20\right.$ e $\left.28^{\circ} \mathrm{C}\right)$, terceiro $\left(30^{\circ} \mathrm{C}\right)$ e quarto $\left(23\right.$ e $\left.25^{\circ} \mathrm{C}\right)$ ínstares, as ninfas que originaram machos apresentaram tendência de duração de desenvolvimento superior àquelas que originaram fêmeas (Tabelas I e II). Todavia, foram verificadas diferenças significativas $(\mathrm{P}<0,05)$ no primeiro $\left(25^{\circ} \mathrm{C}\right)$, segundo $\left(25^{\circ} \mathrm{C}\right)$, terceiro $\left(23^{\circ} \mathrm{C}\right)$ e quinto $\left(28^{\circ} \mathrm{C}\right)$ ínstares. Por outro lado, independentemente dos sexos, a duração relativa dos estágios imaturos de $G$. torresi foi semelhante nas cinco temperaturas estudadas; assim, os períodos de incubação e ninfais de primeiro, segundo, terceiro, quarto e quinto ínstar, representaram, respectivamente, 32-35; 13-16; 9-11, 7-13, 12-13 e $20-23 \%$ do total dos estágios imaturos. Observa-se que as variações são mais acentuadas no terceiro ínstar e podem ser atribuídas ao rápido desenvolvimento biológico ocorrido nesse ínstar a $30^{\circ} \mathrm{C}$.

Com exceção do terceiro ínstar, a duração dos demais estágios imaturos de $G$. torresi foi cerca de duas vezes e meia menor a $30^{\circ} \mathrm{C}(12,47$ dias $)$ que a $20^{\circ} \mathrm{C}(31,66$ dias $)$ tanto para fêmeas como para machos (Tabela II) e independente do ínstar, as ninfas permanecem agregadas próximas às posturas.

Os adultos são brancos logo após a emergência, tornandose acinzentados com o enrijecimento do novo tegumento, sendo facilmente reconhecidos pelo aspecto reticulado da superfície dorsal das asas; a cabeça, as expansões laterais do tórax e as asas apresentam, em geral, um padrão de linhas elevadas que delimitam áreas membranosas e alveoladas nas depressões. As fêmeas são bastante semelhantes morfologicamente aos machos, sendo diferenciadas pela presença do ovipositor. As proporções sexuais de $G$. torresi variaram com a temperatura, sendo as proporções de progênie fêmea menores a 20, 23 e $30^{\circ} \mathrm{C}$, ou seja, 1 fêmea: 1,19 machos; 1 fêmea: 1,31 machos e 1 fêmea: 1,33 machos respectivamente, e maiores a 25 e $28^{\circ} \mathrm{C}$, ou seja, 1 fêmea: 0,84 machos e 1 fêmea: 0,5 machos, respectivamente. LAUGÉ (1985) afirmou que a razão sexual dos insetos pode ser alterada quando submetidos a condições desfavoráveis, temperaturas extremas e/ou escassez de alimento. Portanto, essa predominância de fêmeas nas temperaturas de 25 e $28^{\circ} \mathrm{C}$, indica, provavelmente, que as temperaturas situadas na faixa de 25 e $28^{\circ} \mathrm{C}$ são mais favoráveis ao desenvolvimento de $G$. torresi .

Os valores relativos à duração dos períodos de préoviposição, oviposição, pós-oviposição e longevidade de $G$. torresi, foram inversamente proporcionais à temperatura (Tabela III). A $20^{\circ} \mathrm{C}$, as fêmeas necessitaram aproximadamente de oito dias para iniciar a postura, enquanto que a $30^{\circ} \mathrm{C}$ necessitaram de quatro dias. Independente da temperatura e da atividade sexual, a longevidade dos machos foi sempre maior que a das 
Tabela IV. Temperatura-base ( $\mathrm{Tb})$, constante térmica $(\mathrm{K})$, intercepta (a), coeficiente angular (b) da equação de regressão da velocidade de desenvolvimento dos estágios imaturos de fêmeas e machos de G. torresi, em algodoeiro. Campina Grande, 1999(1).

\begin{tabular}{|c|c|c|c|c|c|c|c|}
\hline Fase & Estágio & $\mathrm{Tb}\left({ }^{\circ} \mathrm{C}\right)$ & $\mathrm{K}(\mathrm{GD})$ & $a \pm E P$ & $\mathrm{~b} \pm \mathrm{EP}$ & $P$ & $\mathrm{R}^{2}$ \\
\hline & & & & Fêmea & & & \\
\hline \multirow[t]{6}{*}{ Ovo } & - & 14,9 & 63,8 & $-0,232980 \pm 0,044199$ & $0,015664 \pm 0,001736$ & 0,0030 & 0,96 \\
\hline & Primeiro & 09,6 & 39,1 & $-0,244458 \pm 0,231877$ & $0,025578 \pm 0,009112$ & 0,0670 & 0,72 \\
\hline & Segundo & 14,6 & 19,0 & $-0,768097 \pm 0,152340$ & $0,052549 \pm 0,005986$ & 0,0030 & 0,96 \\
\hline & Terceiro & 16,7 & 14,5 & $-1,154398 \pm 0,686443$ & $0,069138 \pm 0,026974$ & 0,0830 & 0,78 \\
\hline & Quarto & 15,1 & 22,2 & $-0,681410 \pm 0,189705$ & $0,044995 \pm 0,007454$ & 0,0090 & 0,92 \\
\hline & Quinto & 11,4 & 55,2 & $-0,205543 \pm 0,023988$ & $0,018114 \pm 0,000942$ & 0,0001 & 0,99 \\
\hline Ninfal & - & 13,3 & 146,6 & $-0,090967 \pm 0,020803$ & $0,006820 \pm 0,000817$ & 0,0040 & 0,96 \\
\hline Ovo a adulto & - & 13,9 & 209,4 & $\begin{array}{c}-0,066460 \pm 0,013795 \\
\text { Macho }\end{array}$ & $0,004775 \pm 0,000542$ & 0,0030 & 0,96 \\
\hline \multirow[t]{6}{*}{ Ovo } & - & 14,9 & 63,8 & $-0,232980 \pm 0,044199$ & $0,015664 \pm 0,001736$ & 0,0030 & 0,96 \\
\hline & Primeiro & 09,3 & 40,6 & $-0,228006 \pm 0,250092$ & $0,024623 \pm 0,009827$ & 0,0870 & 0,68 \\
\hline & Segundo & 13,0 & 22,0 & $-0,593486 \pm 0,287235$ & $0,045523 \pm 0,011287$ & 0,0270 & 0,84 \\
\hline & Terceiro & 18,3 & 11,9 & $-1,539149 \pm 0,915442$ & $0,084338 \pm 0,035973$ & 0,1010 & 0,65 \\
\hline & Quarto & 11,9 & 31,6 & $-0,376436 \pm 0,071428$ & $0,031690 \pm 0,002806$ & 0,0010 & 0,98 \\
\hline & Quinto & 12,0 & 53,2 & $-0,225650 \pm 0,057197$ & $0,018799 \pm 0,002247$ & 0,0040 & 0,96 \\
\hline Ninfal & - & 12,9 & 156,9 & $-0,082267 \pm 0,024992$ & $0,006374 \pm 0,000982$ & 0,0070 & 0,93 \\
\hline Ovo a adulto & - & 13,6 & 220,3 & $-0,061595 \pm 0,015785$ & $0,004539 \pm 0,000620$ & 0,0050 & 0,95 \\
\hline
\end{tabular}

${ }^{(1)} 1 / \mathrm{D}=(\mathrm{a} \pm \mathrm{EP})+(\mathrm{b} \pm \mathrm{EP}) \mathrm{t}$, onde $\mathrm{D}$ : duração (em dias) e t: temperatura $\left({ }^{\circ} \mathrm{C}\right) ; \mathrm{EP}=$ erro padrão; $P$ : nível de probabilidade (teste " $\mathrm{t}$ ").

fêmeas (Tabela III). NeAL JR. \& Douglass (1988, 1990) constataram comportamento semelhante para casais de $S$. pyreoides e $C$. cydoniae, cujos machos duravam mais que as fêmeas. Portanto, essas variações de longevidade entre os sexos, podem ser atribuídas a diferentes necessidades de alocação de energia para a atividade de oviposição nas fêmeas.

A fecundidade de $G$. torresi foi maior nas menores temperaturas, variando de 219,3 ovos a $20^{\circ} \mathrm{C}$ até 61,0 ovos a $30^{\circ} \mathrm{C}$, concordando com resultados já relatados para C. cydoniae (Neal JR. \& Douglass 1990). Por outro lado, a $25^{\circ} \mathrm{C}$ a fecundidade média de $G$. torresi foi duas vezes maior que a fecundidade média de G. sanchezi (Schoonhoven et al. 1975) em folhas de feijoeiro. Desta forma, é provável que essa variação tenha ocorrido devido às diferenças entre as espécies de tingídeos e pelo tipo de substrato utilizado pelos autores. $\mathrm{O}$ número médio de ovos/fêmea/dia variou de 5,9 a 3,1, nas temperaturas de 28 e $30^{\circ} \mathrm{C}$, respectivamente.

A velocidade de desenvolvimento para as fases de ovo, ninfa e de ovo à emergência de adultos de G. torresi, mostrouse linear e positivamente relacionada com a temperatura, entre 20 e $30^{\circ} \mathrm{C}$; os valores da temperatura-base para machos e fêmeas foram semelhantes (Tabela IV). Dentro desse limite e para ambos os sexos, ninfas de terceiro ínstar apresentaram maior velocidade de desenvolvimento, o que pode ser confirmado pelos maiores coeficientes angulares das equações de regressão estimados para esse ínstar. Higley et al. (1986) afirmaram que, dentro de certos limites, temperaturas mais elevadas produzem taxas de desenvolvimento maiores, porque as reações bioquímicas se processam mais rapidamente.

As constantes térmicas para as fases de ninfa e de ovo à emergência de adultos machos de $G$. torresi foram superiores às que originaram fêmeas. Para o desenvolvimento ninfal de indivíduos que originaram fêmeas e machos de G. torresi, foram requeridos 146,6 e 156,9 graus-dia, respectivamente, acima dos limites inferiores de 13,3 e $12,9^{\circ} \mathrm{C}$ (Tabela IV). Do mesmo modo, para o desenvolvimento de ovo à emergência de adultos fêmeas e machos de $G$. torresi, foram requeridos 209,4 e 220,3 grausdia, respectivamente, acima dos limites inferiores de 13,9 e $13,6^{\circ} \mathrm{C}$ (Tabela IV). As temperaturas-base obtidas no presente trabalho se assemelham aos resultados de $13,3,14,9$ e $14,3^{\circ} \mathrm{C}$ (BRAMAN \& Pendley 1993), 10,2, 12,2 e 11,2 (Braman et al. 1992) obtidos, respectivamente, para as fases de ovo, ninfal e combinação dos estágios imaturos de $C$. cydoniae e $S$. pyrioides. Por outro lado, os valores da constante térmica obtidos foram inferiores aos valores de $162,9,157,3$ e 318,2 obtidos por BRAMAN \& Pendley (1993) para a constante térmica da fase ninfal e combinação dos estágios imaturos de C. cydoniae, assim como, aos valores de 213,1; 179,2 e 394,0 obtidos por BRAMAn et al. (1992) para a constante térmica da fase ninfal e combinação dos estágios imaturos de $S$. pyrioides. Esses resultados demonstram que $G$. torresi produz maior número de gerações durante o ano e apresenta limites térmicos de sobrevivência semelhantes aos de $C$. cydoniae e $S$. pyrioides. Portanto, em regiões de temperaturas elevadas, semelhantes àquelas encontradas no Nordeste, principalmente nas regiões Central e Centro-Sul do estado do Ceará, onde sua ocorrência tem sido registrada, essa praga apresenta alto potencial de crescimento populacional.

Os resultados obtidos nesse trabalho podem ser utilizados em modelos matemáticos na determinação do número 
de gerações e previsão de populações de G. torresi, em diferentes regiões cotonícolas do Brasil.

Agradecimentos. Ao Dr. Luiz Antônio Alves Costa pela identificação da espécie, cujos exemplares encontram-se depositados no Museu Nacional, Departamento de Entomologia, da Universidade Federal do Rio de Janeiro.

\section{REFERÊNCIAS}

Aldrich, J. R; J. W. Neal JR.; J. E. Oliver \& W. R. Lusby. 1991. Chemistry vis-a-vis maternalism in lace bugs (Heteroptera: Tingidae): alarm pheromones and exudate defense in Corythucha and Gargaphia species. Journal of Chemical Ecology 17: 2307-2322.

Bleicher, E. \& E. C. Freire. 1980. Manejo das pragas do algodoeiro no Estado da Bahia. Campina Grande, EMBRAPA-CNPA, (Boletim Técnico, 6), $16 \mathrm{p}$.

Braman, S. K. \& A. F. Pendley. 1993. Temperature, photoperiod, and agregation effects on development, diapause, reproduction, and survival in Corythucha cydoniae (Heteroptera: Tingidae). Journal of Entomological Science 28(4): 417-426.

Braman, S. K.; A. F. Pendley; B. Sparks \& W. G. Hudson. 1992. Thermal requirements for development, popultion trends, and parasitism of azalea lace bug (Heteroptera: Tingidae). Journal of Economic Entomology 85(3): 870-877.

Costa Lima, A. DA. 1940. Insetos do Brasil. Rio de Janeiro, Escola Nacional de Agronomia, v. 2, cap. 22. (Série didática, 3).

Drake, C. \& E. J. Hambleton. 1934. Brazilian Tingidae (Hemiptera) (Part I). Revista de Entomologia 4(4): 435-451.

Dunbar, D. M. 1974. Bionomics of the andromeda lacebug, Stephanitis takeyai. In: Memoirs Connecticut Entomological Society $\mathbf{2 5}^{\text {th }}$ Anniversary. 277-289.

Hardin, M. R. \& D. W. Tallamy. 1992. Effect of predators and host phenology on the maternal and reproductive behaviors of Gargaphia lace bugs (Hemiptera: Tingidae). Journal of Insect Behaviour 5 (2): 177-192.

Higley, L. G.; L. P. Pedigo \& K. R. Ostlie. 1986. Degday: A program for calculating degree-days, and assumptions behind the degree-day approach. Environmental Entomolology 5: 999-1005.

Horn, K. F.; C. G. Wright \& M. H. FARRIER. 1980. Bionomies of Corythucha associata Osborn and Drake. Journal Ga. Entomol. Society 15: 317-327.

Kearns, R. S. \& R. T. Yамамото. 1982. Maternal behavior and alarm response in the eggplant lace bug Gargaphia solani Heidemann (Tingidae: Heteroptera). Psyche 88: 215-230.

LaUgé, G. 1985. Sex determination: genetic and epigenetic factors, p. 295-318. In: G.A. Kerkut \& L.I.Gilbert. (eds). Comprehensive insect physiology, biochemistry, and pharmacology. Oxford, Pergamon.

Monaco, E. L.; D. W. Tallamy \& R. K. Johnson. 1998. Chemical mediation of egg dumping in the lace bug Gargaphia solani Heidemann
(Heteroptera: Tingidae). Animal Behaviour 56: 1491-1495.

Monte, O. 1939. Lista preliminar dos Tingitidios de Minas Gerais. Revista da Sociedade Brasileira de Agronomia 2: 1-25.

Moraes, G. J. DE. 1981. Ácaros e insetos associados a algumas culturas irrigadas do submédio São Francisco. Petrolina, EMBRAPA-CPATSA (Circular Técnica, 4), $32 \mathrm{p}$.

MoRAES, G. J. DE. 1982. Insetos e ácaro associados a algumas culturas na região de Ouricuri, PE. Petrolina, EMBRAPA-CPATSA (Boletim de Pesquisa 15), $36 \mathrm{p}$.

Moraes, G. J. DE \& F. S. Ramalho. 1980. Alguns insetos associados a Vigna unguiculata Walp. no Nordeste. Petrolina, EMBRAPACPATSA, (Boletim de Pesquisa 1), $16 \mathrm{p}$.

Neal JR., J. W. \& L. W. Douglass. 1988. Development, oviposition rate, longevity, and voltinism of Stephanitis pyrioides (Heteroptera: Tingidae), an adventive pest of azalea, at three temperatures. Environmental Entomology 17(5): 827-831.

Neal JR., J. W. \& L. W. Douglass. 1990. Seasonal dynamics and the effect of temperature in Corythucha cydoniae (Heteroptera: Tingidae). Environmental Entomology 19(5): 1299-1304, 1990.

Olckers, T. 2000. Biology, host specificity and risk assessment of Gargaphia decoris, the first agent to be released in South Africa for the biological control of the invasive tree Solanum mauritianum. Biocontrol 45: 373-388.

Rogers, C. E. 1977. Laboratory biology of a lace bug on sunflower. Annals of the Entomological Society of America 70: 144-145.

Schoonhoven, A. Van; F. Burbano \& R. ARenas. 1975. Notes on the biology of the lace bug Gargaphia sanchezi (Hemiptera, Tingidae) a pest of beans (Phaseolus vulgaris). Turrialba 25: 327.

Sheeley, R. H \& T. R. Yonke. 1977. Biological notes on seven species of Missouri tingids (Hemiptera: Tingidae). Journal of the Kansas Entomological Society 50: 342-356.

Silva, C. C. A. DA \& S. M. De L. Barbosa. 1986. Surto do percevejo de renda, Gargaphia torresi, na cultura do feijão em Alagoas. Pesquisa Agropecuária Brasileira 21: 1003-1004.

Silverman, J. \& R. D. Goeden. 1979. Life history of the lacebug, Corythucha morrilli Osborn and Drake, on the ragweed, Ambrosia dumosa (Gray) Payne, in Southern California (Hemiptera-Heteroptera: Tingidae). Pan-Pacific Entomologist 55: 305-308.

SLANSKY JR. F. 1980. Quantitative food utilization and reproductive allocation by adult milkweed bugs, Oncopeltus fasciatus. Physiological Entomology 5: 73-86.

Tallamy, D. W. 1985."Egg dumping" in lace bugs (Gargaphia solani, Hemiptera: Tingidae) Behavioral Ecology and Sociobiology 17: 357-362.

Tallamy, D. W \& L. A. Horton. 1990. Costs and benefits of the eggdumping alternative in Gargaphia lace bugs (Hemiptera: Tingidae). Animal Behaviour 39: 352-360.

Tallamy, D. W. \& R. F. Denno. 1981. Maternal care in Gargaphia solani (Hemiptera: Tingidae). Animal Behaviour 29: 771-778.

Vieira, F. V.; J. H. R. Santos; I. T. Lima \& P. E. F. Castro. 1991. Influência do "mosquito" do algodoeiro, Gargaphia torresi Lima, 1922 (Hemiptera, Tingidae), sobre a produção do algodoeiro anual. Ciência Agronômica 22(1/2): 71-76.

Vogt, T. E. \& J. E. 1986. Mcpherson. Life history and laboratory rearing of Corythucha juglandis (Hemiptera: Tingidae) with descriptions of immature stages. Great Lakes Entomologist 19: 221-233.

Recebido em 10.III.2004; aceito em 30.VIII.2004 